\title{
LAS PROMESAS ELECTORALES DE LOS CANDIDATOS A LA PRESIDENCIA DE LA REPÚBLICA DE COSTA RICA, DIVULGADAS EN MEDIOS DE PRENSA ESCRITA, 2014
}

\section{ELECTORAL PROMISES OF CANDIDATES TO THE PRESIDENCY OF THE REPUBLIC OF COSTA RICA, PUBLISHED IN WRITTEN MEDIA, 2014}

\author{
Yagnna Nicolás Jiménez ${ }^{*}$ \\ Edwin Alvarado Mena* \\ Jeff Rodríguez Alvarado* \\ Allan Abarca Rodríguez ${ }^{* * * *}$
}

\section{RESUMEN}

\begin{abstract}
Este artículo es el resultado de una investigación de seguimiento a la campaña electoral 2014, en Costa Rica. En las 350 ediciones impresas de los medios de prensa escrita La Nación, Diario Extra y La Teja, correspondientes a la campaña electoral 2014 (período comprendido entre el 2 de octubre de 2013 y el $1^{\circ}$ de febrero de 2014) se divulgan 1417 promesas electorales como parte de crónicas, reportajes o entrevistas realizadas a los candidatos a la Presidencia de la República.
\end{abstract}

PALABRAS CLAVE: COSTA RICA * ELECCIONES * POLÍTICOS * PUBLICACIÓN PERIÓDICA * PLAN DE DESARROLLO

\footnotetext{
* Realizando pasantía en el Instituto Latinoamericano de Naciones Unidas para la prevención del delito y la atención del delincuente (ILANUD), Costa Rica. yagnna2691@gmail.com

** Cursando la maestría en Políticas Públicas, Flacso-Ecuador. ealvaradoucr@gmail.com

*** Ministerio de Planificación y Política Económica (MIDEPLAN), Costa Rica jeff.r.ucr@hotmail.com

Escuela de Ciencias Políticas y Escuela de Salud Pública, Universidad de Costa Rica. allan.abarca@ucr.ac.cr
} 


\section{ABSTRACT}

This article is the result of an investigation that followed up the presidential elector campaign 2014, Costa Rica. In the 350 printed editions of La Nación, Diario Extra and La Teja papers, which were published during the 2014 presidential campaign (from October 2nd 2013 to February 1st 2014) a total of 1417 electoral promises were made as part of chronicles, stories and interviews to candidates to the Presidency of the Republic of Costa Rica.

KEY WORDS: COSTA RICA * ELECTIONS * POLITICS * NEWSPAPERS * DEVELOPMENT PLANS

\section{INTRODUCCIÓN}

Un Programa de Gobierno, integralmente concebido, contiene principios (referentes axiológicos) y la constitución de una oferta electoral que en el mejor de los casos, incluye una clara reseña de los problemas públicos, las metas/objetivos perseguidos - concebidos como promesas-y el cómo se lograrán. Por el contrario, una perspectiva limitada de un Programa de Gobierno se reduce a un listado de propósitos o de promesas.

Los partidos políticos —en su disputa por la Presidencia de la República - en cualquiera de los dos tipos de Programa de Gobierno mencionados, asumen un conjunto de promesas promocionadas a lo largo de la campaña electoral constitutivas de la base de su ejercicio de poder en un periodo formal.

Ante la insistencia de los analistas políticos y las autoridades electorales en recalcar que el votante ha de informarse para decidir con sentido crítico por una candidatura en específico, surge obligatoriamente la interrogante acerca del tipo de información que efectivamente recibe.

Kang y Bingham (2010) apuntan que existe una alta correlación entre lo que quieren los ciudadanos y lo que finalmente resulta de los hacedores de las políticas públicas, esto es, entre votantes $y$ posteriores funcionarios de Gobierno. Se genera un dilema para quien ejercerá el Gobierno: prestar atención a las promesas efectuadas en campaña o a las preferencias cambiantes del votante medio; ambas vinculadas estrechamente a la forma cómo se ejercerá el gasto público, o sea, de las políticas públicas a adoptar y su financiamiento.
Para Aragonès, Palfrey y Postlewaite, la relación entre los políticos y los votantes, mediada por las promesas de campaña, genera múltiples equilibrios. Estos autores señalan que "campaign promises affect voters' expectations about what policies will be chosen by an elected official, and they provide a bench mark for voters to link policy decisions with future re-election"1 (2007: 847). No obstante, ese binomio - expectativa y posible castigo del electorado- es particularmente visible en sistemas electorales con reelección continua.

De esta forma, no solo hay que preguntarse sobre qué se promete, las reglas electorales, el sistema de partidos políticos y la cultura política de una sociedad en particular, sino sobre los principios respecto de los cuales los partidos deciden conformar una particular oferta electoral.

Maravall, Przeworski y Casado señalan varios estudios que relacionan el apoyo político ciudadano con sus expectativas de la economía, con lo que los compromisos o promesas sí son tomados en cuenta: "las cuestiones de si la gente percibe con precisión la situación económica, si extrapola el pasado para hacer pronósticos y si permite o no qué creencias $y$ compromisos políticos previos moldeen sus decisiones de voto, son empíricas..." (1999: 13). Existe una racionalidad del votante particularmente reafirmado con promesas en el ámbito de su bienestar económico.

$1 \quad$ “[...] las promesas de campaña afectan las expectativas de los votantes sobre cuáles políticas serán elegidas por un funcionario electo y proporcionan un referente para los votantes que relacionan las decisiones de la política con la reelección futura" (2007: 847). Traducción propia. 
En forma complementaria un proceso electoral podría estar influenciado por el voto temático (óissuevoting); con lo cual los votantes expresarían particular interés en aquellas promesas - de políticas públicas o de actuación política- que refieran a temas de agenda nacional que se introducen como parte de la agenda electoral. Por ejemplo, en Costa Rica se presumió que para la campaña 2014, podría situarse en importancia para el electorado las posiciones de los candidatos sobre la técnica de reproducción sexual asistida "Fertilización In Vitro-fiv" o sobre la legislación en derechos de convivencia para las personas del mismo sexo; ambos temas presentes en la agenda de discusión pública en años recientes.

Ahora bien, independientemente de las reglas del sistema electoral, el procesamiento de la información que realiza el votante podría verse filtrado por sus valores políticos. Para Anduiza y Bosch (2004) si el elector se posiciona ideológicamente podría ser que una promesa electoral determinada la califique según sus consecuencias, reconociendo la posición natural de quien la emite. Por ejemplo, si un candidato ofreciera miles de puestos de trabajo, pero el elector lo caracteriza de derecha, posiblemente esto le haga inferir que la creación de esas fuentes de empleo iría aparejado con una flexibilización de los derechos laborales, lo que pondría en riesgo algunas garantías sociales.

Los valores políticos de la persona también podrían estar en consonancia con la cercanía o identificación natural que siente hacia un determinado partido político, lo que se conoce como el "Modelo Michigan", emergido en los años 70 del siglo xx. En términos de las promesas electorales no solamente cabría esperar la afinidad partidaria del elector sino también cuál es la intensidad percibida de esa relación en unacoyuntura, lo cual puede estar relacionado con el tipo y orientación de las promesas.

Para el "Modelo Racional" hay un elector con capacidad de discernimiento, que recopila, procesa y analiza la información. No obstante, hay problemas evidentes: un déficit sistemático de información, un sobre énfasis en el voto cautivo clientelar por parte de los partidos, una ausencia de mecanismos institucionales que fomenten una ciudadanía fuerte (Máiz, 2006) y una carencia para procesar una información que de por sí es limitada.

Incluso la difuminación ideológica, tanto de electores como de partidos políticos, que certifica una erosión de sus diferencias doctrinarias, implica un acercamiento a lo que se denomina como la "mediana ideológica", que no es más que la estrategia o tendencia a situarse más próximos a la posición de la mayoría ciudadana (Anduiza y Bosch, 2004). De eso se puede inferir que las promesas y los medios o principios para alcanzarlos, no son tan distintos entre una amplia gama de partidos políticos.

En medio de esas teorías es posible que aunque limitada, se dé cierta racionalidad utilitarista del votante que le permita valorar las promesas de campaña; es posible también que la orientación de su voto quede circundada por una identidad social con un partido, cierto reconocimiento de los antecedentes en gobierno y una estimación de la credibilidad y capacidad probable del candidato en cuestión.

Para Keefer (2007), en las democracias jóvenes hay una incapacidad manifiesta de los competidores políticos para que sus promesas sean creíbles, lo que les motiva a preferir las políticas clientelistas. Al respecto, Susan Stokes ha realizado estudios en América Latina que le permite afirmar que en las campañas priman pronunciamientos populistas de candidatos que saben de su futuro incumplimiento, pero que lo anuncian a ciudadanos sin un conocimiento político (cfr. Stokes, 2001; citado en Hernández, 2008: 268). Por esta razón, Hernández afirma que las promesas difíciles de cumplir son propias de "sociedades con bajos niveles de información política" (2008: 268) y que fundamentalmente se da en las promesas de ámbito económico.

De esta manera, a la decisión del votante por una candidatura le subyacen múltiples elementos como la tradición familiar, la valoración sobre el partido gobernante $y$ las expectativas respecto a un candidato. Este último elemento está mediado por la oferta electoral que efectivamente recibe y su forma de valorarla (credibilidad, posible repercusión en su calidad de vida, entre otros). 
Pero la pregunta fundamental es: ¿cuánta correspondencia existe entre el voto informado que se le exige al ciudadano $y$ la posibilidad de este de enterarse al menos de las propuestas programáticas de los candidatos a la Presidencia de la República? Si bien, existe multiplicidad de mecanismos para divulgar propuestas $u$ ofertas políticas (redes sociales, páginas web oficiales, debates, foros, entre otros), lo cierto del caso es que los medios de comunicación masiva tradicionales son esenciales por su amplia cobertura ${ }^{2}$,aunque esto por sí mismo produce restricción en frecuencia y calidad de lo que es capaz de informarse el elector.

El objetivo principal de este artículo es analizar las promesas electorales (de los 13 partidos políticos con candidatos presidenciales inscritos oficialmente) difundidas en tres medios de prensa escrita, durante la campaña presidencial 2014, en Costa Rica: La Nación, Diario Extra y La Teja.

Según el Código Electoral (artículo 149), la campaña electoral se define como la fase comprendida desde el día de la convocatoria a elecciones hasta el día de su realización. Se analiza el universo de publicaciones, compuesto por 123 emisiones del periódico La Nación, 104 emisiones del periódico Diario Extra (que no circula el día domingo) y 123 emisiones del periódico La Teja; todos ellos comprendidos entre el 2 de octubre de 2013, momento de inicio de la campaña y el $1^{\circ}$ de febrero de 2014 , un día previo a las elecciones.

Las unidades de análisis son las crónicas, entrevistas y reportajes; la unidad de registro es el recuento (frecuencia) de las promesas electorales atribuibles al candidato o partido

$2 \quad$ Es interesante que en la encuesta realizada por el Centro de Investigación y Estudios Políticos de la Universidad de Costa Rica (CIEP-UCR), entre el 3 y el 13 de febrero de 2014, luego de las elecciones, la razón "propuesta o programa de gobierno" figura entre las principales razones por las que los votantes justifican haberle dado su adhesión a 4 de los 5 candidatos con mayor cantidad de votos efectivos: Solís (PAC), Villalta (FA), Guevara (ML) y Piza (PUSC). Para una ampliación $c f r$. Centro de Investigación en Estudios Políticos, Universidad de Costa Rica. Informe de la encuesta post-electoral febrero 2014. Estudios de opinión sociopolítica. Universidad de Costa Rica, 2014: 15. político ${ }^{3}$. Finalmente, la unidad categorial de análisis refiere a la materia clasificable de la promesa electoral, en este sentido se agrupan en 25 ejes temáticos.

A partir de la sistematización, se identifica la frecuencia de promesas divulgadas por candidato-medio de prensa y se clasifican según el eje temático.

Tres elementos metodológicos acerca del análisis:

$\diamond \quad$ Hay promesas electorales que son reincidentes en la divulgación de los medios, por ejemplo, "construcción de un metro en la ciudad de San José" o la "generación de 500 mil empleos". El recuento de las promesas divulgadas toma en consideración la frecuencia total, sin importar si la misma promesa se repite en varias ocasiones o no se repite, precisamente porque interesa determinar el volumen $y$ tipo (según eje temático) que podría percibir el lector del medio.

$\diamond \quad$ Es evidente que cada promesa electoral presenta distinto nivel de abstracción, lo cual, eventualmente, incide en su evalualibidad (vg. "reactivar el servicio de tren San José-Limón" o "alcanzar el 1\% del pis para el presupuesto del Ministerio de Cultura" tienen un nivel de concreción/verificabilidad alto a diferencia de "más créditos para que los jóvenes tengan vivienda", el cual es una promesa con verificabilidad baja). Es evidente que en la promesa capturada hay variaciones

$3 \quad$ Los 13 partidos políticos y candidatos partícipes en la contienda política 2014, en Costa Rica, fueron: Johnny Araya Monge (Partido Liberación Nacional-PLN), Luis G. Solís (Partido Acción Ciudadana-PAC), José M. Villalta (Frente AmplioFA), Otto Guevara (Movimiento Libertario-ML), Rodolfo Piza (Partido Unidad Social CristianaPUSC), José M. Corrales (Patria Nueva-PN), Sergio Mena (Partido Nueva Generación-PNG), Carlos L. Avendaño (Partido Restauración-PREN), Wálter Muñoz (Partido Integración NacionalPIN), Óscar López (Partido Accesibilidad Sin Exclusión-PASE), José M. Echandi (Partido Avance Nacional-PAN), Justo Orozco (Partido Renovación Costarricense-PRC) y Héctor Monestel (Partido de los Trabajadores-PT). 
en torno a la presencia/ausencia de explicación del cómo se concretaría cada una, incluso, algunas de estas son redactadas como objetivos. No obstante, para efectos de este recuento no se hizo ninguna taxonomía acerca de la precisión-imprecisión de esas promesas, lo cual pertenecería a otro posible estudio investigativo.

$\diamond \quad$ Cada promesa electoral potencialmente es clasificable en varios temas, aun así, se decide imputarla a uno solo, para esto se privilegia al tema general que el medio de comunicación aborda en su crónica, reportaje o entrevista.

\section{DIVULGACIÓN DE LAS PROMESAS ELECTORALES}

Los partidos políticos pueden ser considerados como "...máquinas que compiten por los votos del electorado para conseguir parcelas de poder. Es decir, en el seno del sistema político el partido obtiene un rendimiento que puede ser medido en votos $y$ en puestos de mando..." (Alcántara, 2004: 81). A partir de tal concepción, se asume que cada agrupación política partidaria optimiza sus recursos y estrategias en aras de sumar votos efectivos que le permitan ganar la contienda electoral.

La captación de votos no resulta una práctica del todo sencilla, debido a que además de intentar proyectarse como la "mejor opción para gobernar", los partidos apuestan por maximizar la adhesión a través de lo que se conoce como la "emisión de ofertas electorales".

La emisión de ofertas representa, en lo fundamental, una especie de ejercicio estratégico partidario que forma parte de la corriente de las comunicaciones persuasivas de la campaña electoral (Canel, 1998). La finalidad de estas es proyectarle al elector aquellas metas, propuestas e ideales que los partidos pretenden concretar en los eventuales escenarios de un gobierno.

Resulta claro que la oferta electoral incluye a las promesas electorales (es su centro neurálgico), aunque no se agota en ellas, por ese motivo en este artículo los investigadores prefieren utilizar el término promesa electoral. La promesa es la expresión de cumplimiento que realiza un político de una obra, política pública o meta, para afectar positivamente a una población o sector, el cual puede tener un alcance local, regional o nacional.

La importancia que ha alcanzado el tema dentro del sistema político ha generado diversas iniciativas para brindarle al elector una sistematización de las promesas de los candidatos. En el caso de la campaña 2014, entre muchas otras se destacan las siguientes:

a) El Tribunal Supremo de Elecciones (TSE), en aras de "promover un votante informado $y$ de ofrecer un espacio igualitario a todos los candidatos a la Presidencia”, dispuso en su sitio web la aplicación "Herramientas para un voto informado". Este incluyó perfiles de los candidatos y un enlace a sitios web de los Programas de Gobierno de los partidos políticos; no obstante, para cuatro partidos políticos no se dispuso de alguna información de programa: el PT, el PPN, PIN y el PAN ${ }^{4}$.

b) En su sitio web, el periódico La Nación generó la herramienta "Palabra de político", con la cual presentó y contrastó promesas de cinco candidatos (Solís (PAC), Araya (PLN), Guevara (мL), Villalta (FA) y Piza (pusc)), para luego calificar cada una de las promesas como "verdadera", "falsa" o "medio verdad", principalmente cuando el candidato anunciaba un problema por atacar o una aspiración. Por ejemplo, el candidato del pac prometió "la universalización del segundo idioma en el sistema educativo del país", pero la evidencia aportada por el medio refiere a que la cobertura de educación bilingüe en primaria es de $87,8 \%$ y de un $100 \%$ en secundaria $y$ por eso, califica la promesa

$4 \quad$ La no aparición de un Programa de Gobierno para estos 4 partidos pudo tener como razón el que tales agrupaciones no contasen con un documento como tal o que presentaron su plan extemporáneamente, lo cual imposibilitó que este se pudiera accesar desde el sitio web oficial del TSE, tal y como sucedió para el caso del PAN. Además, esto evidencia la ausencia de una normativa electoral acerca de un Programa de Gobierno que en determinado tiempo y forma, deban presentar los aspirantes a la Presidencia de la República. 
como una "medio-verdad" (La Nación, 08/01/2014).

c) Otra iniciativa fue la del Estado de la Nación al disponer en su sitio web de un "Sistema de seguimiento de la oferta electoral", que contenía audios de entrevistas realizadas a candidatos a la presidencia, referencias de páginas web de candidato o partidos políticos y un balance de promesas, según desafíos identificados en materia de desarrollo humano.

d) Con el título "Elecciones 2014. Aporte para un voto informado" la Universidad de Costa Rica, presentó en su sitio web un trabajo elaborado por la Oficina de Divulgación e Información (odI), el cual sistematizó propuestas-promesas como parte de foros $y$ debates organizados por instancias del propio centro educativo.

\section{PROMESAS EN PRENSA ESCRITA}

Las referencias explícitas sobre Programas de Gobierno (PG), fueron publicadas por $L a$ Nación, respecto a cinco candidatos a la Presidencia (tabla 1).

\section{TABLA 1}

PRINCIPALES ABORDAJES DEL PERIÓDICO LA NACIÓN SOBRE PROGRAMAS DE GOBIERNO CAMPAÑA ELECTORAL COSTA RICA, 2014

\begin{tabular}{|c|c|c|}
\hline PARTIDO & LANZAMIENTO & ANOTACIONES \\
\hline PLN & $10 / 12 / 2013$ & $\begin{array}{l}\text { El 11/12/2013 se publica crónica sobre el lanzamiento oficial del PG. } \\
\text { El 20/12/2013 se refiere al PG tanto en primera plana como en un amplio reportaje. El } \\
\text { medio asegura que promesas sobre "política social" es lo que prima. Al día siguiente } \\
\text { una crónica señala que el déficit fiscal afectaría el posible cumplimiento de tales } \\
\text { promesas. }\end{array}$ \\
\hline ML & $13 / 01 / 2013$ & $\begin{array}{l}\text { El medio publica una extensa crónica el } 21 / 01 / 2014 \text { destacando que la flexibilidad en } \\
\text { los requisitos para iniciar negocios era el punto central de la propuesta. }\end{array}$ \\
\hline PAC & $11 / 12 / 2013$ & $\begin{array}{l}\text { El 12/12/2013 se emite crónica sobre el lanzamiento oficial del PG. } \\
\text { El 25/12/2013 un amplio reportaje sobre el PG. Resalta que el énfasis está en el } \\
\text { combate contra la corrupción. }\end{array}$ \\
\hline $\mathrm{FA}$ & $28 / 11 / 2013$ & $\begin{array}{l}\text { El 29/11/2013 se publica una amplia crónica sobre el lanzamiento oficial del PG. } \\
\text { El 17/12/2013 tanto en primera plana como en un reportaje, se destaca un regreso } \\
\text { al Estado regulador y benefactor. El editorial de esa fecha critica lo planteado; al } \\
\text { día siguiente, también, aparece una crónica que destaca elementos negativos de la } \\
\text { propuesta. }\end{array}$ \\
\hline PUSC & $17 / 12 / 2013$ & $\begin{array}{l}\text { El 18/12/2013 se emite crónica sobre el lanzamiento oficial del PG. } \\
\text { El 28/12/2013 se publica un amplio reportaje. Se asegura que el énfasis del PG gira en } \\
\text { torno a fomentar el desarrollo de las regiones del país a partir de sus condiciones. }\end{array}$ \\
\hline
\end{tabular}

$P G$ : Programa de Gobierno.

Fuente: $\quad$ Elaboración propia basado en la revisión de 123 ediciones del periódico La Nación (2 de octubre de 2013 - $1^{\circ}$ febrero de 2014).

Los otros medios — La Teja y Diario Extra- publicaron crónicas sobre las emisiones del Programa de Gobierno (vg. La Teja, 11 de diciembre de 2013, anuncia el PG del PLN) pero lo hace sin profundizar el tema.

El periódico La Nación también publicó crónicas especiales sobre las promesas de campaña en general o puntualizando promesas de 3 o de 5 candidatos sobre determinados tópicos:

$\diamond \quad$ El 29 de octubre de 2013 realizó una crónica especial de las promesas en torno a las finanzas, la gestión y la atención médica de la Caja Costarricense de 
Seguro Social-ccss (candidatos Araya, Villalta, Guevara, Solís y Piza).

$\diamond \quad$ El 10 de diciembre de 2013, reportaje especial en que destaca que los candidatos prometen pero sin decir el cómo lograrán sus cometidos.

$\diamond \quad$ El 3 de diciembre de 2013, en la primera página se destaca las prioridades de un eventual Gobierno de Araya, Villalta o Guevara, asimismo, se publica el resultado de una entrevista con ellos. El medio destaca esos 3 nombres en virtud de que la encuesta unimer del 1 de diciembre de 2013, refería un aparente triple empate en las preferencias electorales.

Es necesario precisar que las promesas electorales están ubicadas en las crónicas especiales sobre los Programas de Gobierno, pero también hay referencias de promesas como producto del registro realizado por el medio informativo de las giras de los candidatos, discursos en plazas públicas, crónicas sobre debates y entrevistas particulares.

Un análisis exhaustivo de las 123 ediciones del periódico La Nación, correspondientes al periodo en estudio, indica que en 67 de estas $(54,5 \%)$ fueron divulgadas promesas de campaña de al menos un candidato. Este es el medio escrito que mayor número de ediciones con promesas presenta, ya que solamente en el $31,7 \%$ de las ediciones del Diario Extra y en el 10,6\% de La Teja, se presenta al menos una promesa de un candidato a la presidencia.

La Nación también fue el medio que registró mayor cantidad de promesas y las divulgó: 1190 promesas electorales de los 13 candidatos a la Presidencia de la República. La diferencia es amplia en comparación con los otros medios: Diario Extra divulga 190 y La Teja apenas 37.

En el periódico La Nación y en el Diario Extra el candidato Araya (PLN) fue el postulante a la presidencia con más ediciones en las que al menos fuera referenciada una promesa suya (cuadro 1).
CUADRO 1

PROPORCIÓN DE EDICIONES EN LA NACIÓN Y

EL DIARIO EXTRA SEGÚN 5 CANDIDATOS CON MAYOR VOTO EN LAS ELECCIONES

CAMPAÑA ELECTORAL, COSTA RICA, 2014

\begin{tabular}{ccc}
\hline PARTIDO POLÍTICO & LANACIÓN & DIARIO EXTRA \\
\hline PLN & $38,8 \%$ & $14,4 \%$ \\
FA & $36,4 \%$ & $12,5 \%$ \\
PAC & $33,9 \%$ & $9,6 \%$ \\
ML & $33,1 \%$ & $11,5 \%$ \\
PUSC & $26,4 \%$ & $10,6 \%$ \\
\hline
\end{tabular}

Fuente: Elaboración propia con base en la revisión de 227 emisiones del Diario Extra y La Nación (periodo 2 de octubre $2013-1^{\circ}$ febrero de 2014).

Abordando el tema desde el punto de vista cuantitativo, la diferencia de información sobre promesas entre los primeros cinco candidatos el día de las elecciones no es relevante; lo que es destacable es la diferencia entre los medios para divulgar promesas o propuestas de campaña.

La baja frecuencia registrada en La Teja hace irrelevante ofrecer el dato desagregado por partido político.

\section{PROMESAS Y CANDIDATOS}

El día de las elecciones, 2 de febrero de 2014, cinco candidatos (Araya, Solís, Villalta, Guevara y Piza) registran el 95\% de la votación para Presidente de la República. La única coincidencia entre las encuestas divulgadas (en todo el período y por todos los medios de comunicación)se dio en que de la totalidad de candidatos en contienda, esos cinco eran los de mayor intención de voto, de ahí que no sea de extrañar que esos partidos políticos fueran los privilegiados para que los diarios nacionales divulgaran sus promesas.

En el periódico La Teja, casi una tercera parte de las promesas publicadas corresponden a Otto Guevara $(32,4 \%)$, seguido de Solís, Araya y Piza casi con una quinta parte cada uno $(21,6 \%, 18,9 \%$ y $18,9 \%$ respectivamente). 
El candidato del Frente Amplio apenas registra un $8,1 \%$. Pero las diferencias entre los candidatos no son significativas debido a la baja cantidad de las menciones de promesas en este medio.

Publicaciones tanto de La Nación como del Diario Extra evidencian que el candidato del pLn encabeza las menciones de promesas electorales: $25,8 \%$ y 21,1\%: se recalca que en La Nación los candidatos Villalta, Solís y Guevara se mantienen aproximados en las menciones de promesas $(20,3 \%, 18,3 \%$ y $18,2 \%)$, mientras que en Diario Extra son Guevara y Solís (14,2\% y 13,2\%) quienes se presentan en la segunda $y$ tercera posición en la frecuencia de menciones (gráfico 1).

\section{PROMESAS ELECTORALES DIVULGADAS EN LA NACIÓN Y EN EL DIARIO EXTRA PARA CADA CANDIDATO EN LA CAMPAÑA ELECTORAL \\ COSTA RICA, 2014 \\ (EN PORCENTAJE)}
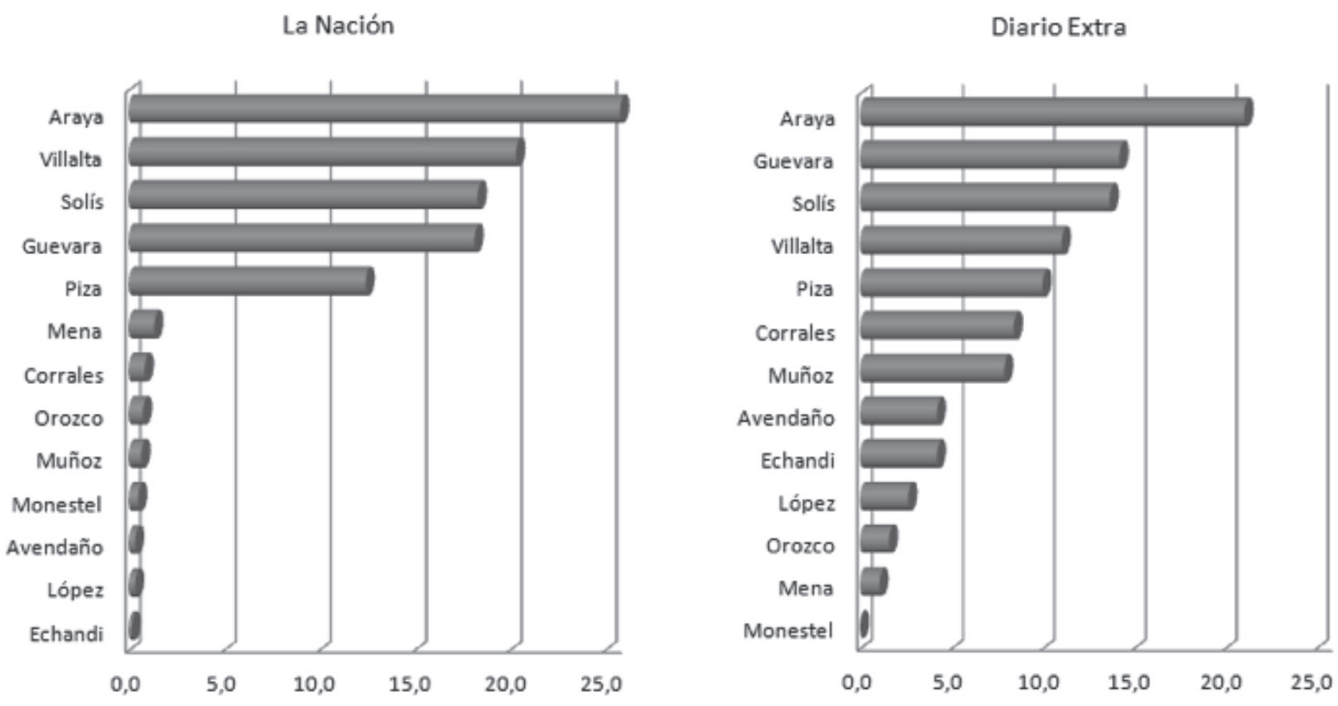

Fuente: Elaboración propia con base en la revisión de 227 emisiones de Diario Extra y La Nación (período 2 de octubre de $2013-1^{\circ}$ de febrero de 2014).

\section{CANDIDATURAS MARGINADAS}

Del total de promesas electorales que da a conocer $L a$ Nación, tan solo el $5 \%$ se atribuyen a los 8 candidatos que precisamente, obtuvieron menor cantidad de votos en las elecciones presidenciales: Corrales, López, Orozco, Avendaño, Echandi, Monestel, Mena y Muñoz. El periódico La Teja no registra mención siquiera de una promesa electoral de esos 8 candidatos.

Esa limitada difusión ¿es aceptable, en tanto la baja intención de voto que para esos candidatos las encuestas evidenciaron en el inicio de la campaña política? o ¿es censurable en tanto repercusión negativa al sistema democrático electoral, que limita al electorado conocer lo más básico de un partido como es su oferta electoral? Es válido plantearse la pregunta en medio de una discusión más amplia que abarque aspectos como: financiamiento a los partidos políticos, validez de las encuestas, libertad del medio a publicar, equidad en la información, estrategias de los 
partidos para dar a conocer sus promesas, entre otros.

La divulgación de promesas electorales de los ocho candidatos con menor votación en el Diario Extra alcanzó el 29,9\%.Se destaca que de todas las promesas divulgadas por este medio, el 8,4\% fue del candidato José M. Corrales (pN) y el 7,9\% de Wálter Muñoz (Pin), lo cual indica que por lo menos en el apartado de dar a conocer la oferta de promesas electorales, este medio fue inclusivo.

\section{PROMESAS SEGÚN TEMAS}

Luego de un análisis exhaustivo, el equipo investigador agrupó en 25 temas las promesas electorales divulgadas. En el periódico La Nación la agenda de promesas electorales la encabezó "educación", la "política fiscal" y "ambiente/energía” (gráfico 2).
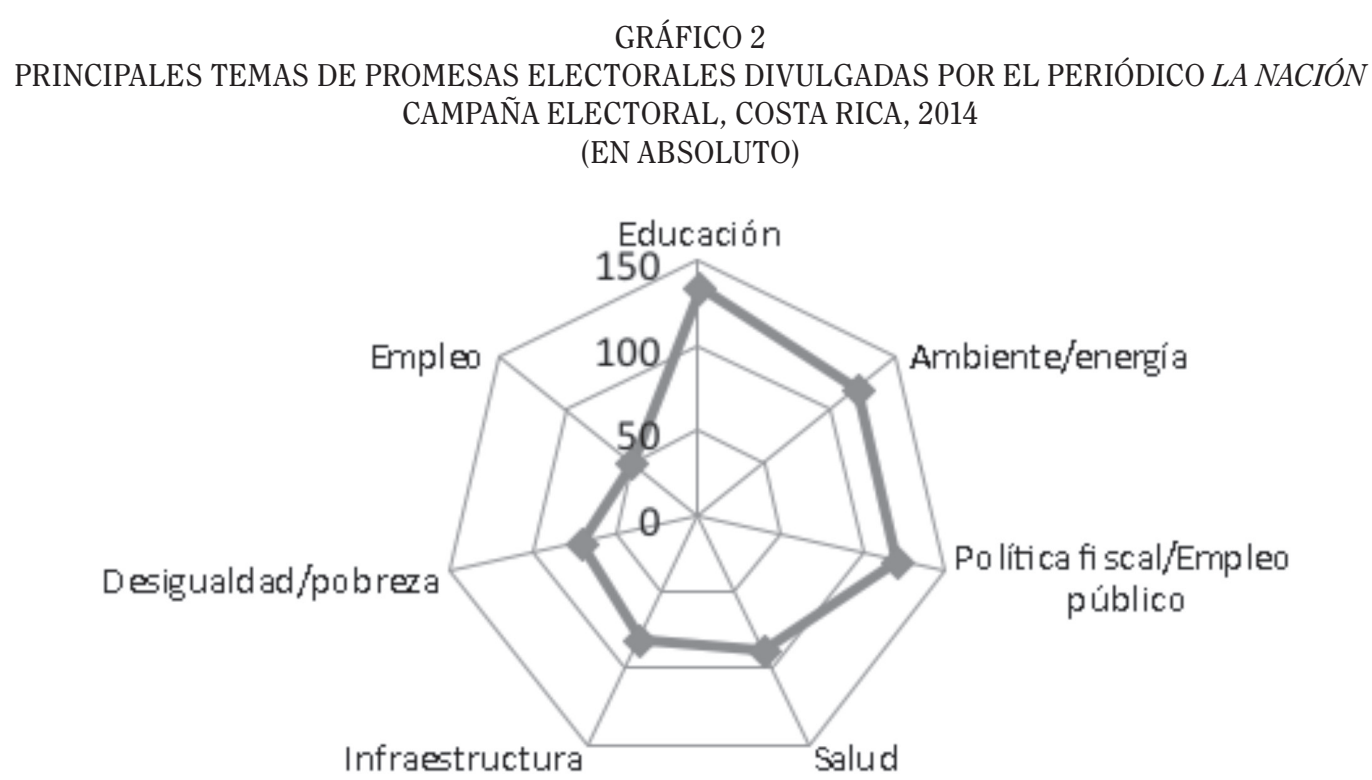

Fuente: Elaboración propia con base en la revisión de 123 ediciones del periódico La Nación (2 de octubre de 2013 - $1^{\circ}$ de febrero de 2014).

El hecho de que un tema domine las promesas electorales que se divulguen no significa necesariamente que sea un tema conflictivo en la agenda nacional. Las promesas entorno a mejorar el acceso y la calidad de la educación nacional alcanzaron las 135 promesas divulgadas, sin que fuera un tema polémico o generador de debates importantes.

Es destacable que el tema "fiscal" se posicionó en el segundo lugar, con 120 menciones, el cual involucra promesas en los ámbitos: empleo público, regulaciones de salarios $y$ alcances de una eventual reforma fiscal.
Un segundo lugar lo ocupa el tema "ambiente" con 120 promesas, pero la mayoría de los elementos ligados a este se dieron en el marco de la producción energética, el acceso al agua y la protección ambiental.

La "salud" y la "infraestructura" se posicionaron entre los temas prioritarios de promesas electorales - puestos 4 y 5 - con 88 y 81 promesas respectivamente, lo cual es entendible en el marco de la discusión del deterioro de las vías y la modernización de los puertos, además de la denominada crisis de los servicios de salud que presta la ccss; esos dos temas han 
estado vigentes en la agenda nacional de problemas en los últimos años.

Cabe destacar otros temas que fueron sensibles en cuanto a frecuencia de promesas divulgadas, por ejemplo: combate a la pobreza (72), empleo (51), seguridad ciudadana (50), agricultura (49), corrupción (44) y producción (44).

La "seguridad ciudadana" no fue tema principal de debate y la mayoría de las promesas al respectose referían a políticas penitenciarias (cárceles).

El tema "empleo"como generador de promesas de campaña fue relevante, máxime por un asunto de coyuntura: según el Instituto Nacional de Estadísticas y Censos (INEC), a julio del 2013, el desempleo abierto es del 8,5\% lo que habla del deterioro al respecto, ya que en el año 2012 alcanzó el 7,8\% y en el 2011, el 7,7\%, además que el candidato Guevara (мL) trató de colocarlo en la agenda desde el propio inicio de su campaña política.

El Diario Extra refiere que los cinco temas en que se agrupan la mayoría de promesas electorales divulgadas son los siguientes:

1. Solvencia/eficiencia del Estado (24).

2. Política fiscal/empleo público (22).

3. Infraestructura (20).

4. Educación (17).

5. Empleo (17).

El número reducido de promesas divulgadas por La Teja (37) hace irrelevante una clasificación, pero al menos cabe señalar que las de mayor frecuencia se concentraron en el tema "combate a la corrupción" (5).

\section{CONCLUSIONES}

Las promesas electorales que los medios La Nación, Diario Extra y La Teja divulgan son producto de: a) crónicas realizadas a partir de la revisión de los programas de gobierno, b) la recopilación de promesas realizadas por los candidatos en giras políticas o en la participación en foros de diversa índole y c) lo esbozado por candidatos en entrevistas o reportajes que el propio medio realiza.
Se revela que durante la campaña electoral aproximadamente, 1200 promesas electorales fueron publicadas por el periódico $L a$ Nación, de las cuales el pun encabezó la lista (307), seguida del FA (242), PAC (218), ML (216) y pusc (148).Del resto de ocho agrupaciones políticas, apenas se registró un $5 \%$ de las promesas; por ejemplo, del pan se publicaron 2 promesas, de PREN $y$ PASE solamente 4.

El Diario Extra publica 190 promesas, la mayoría del PLN (40), seguido del mL (27) y del pac (26), aunque cabe destacar que publicó mayor información de promesas electorales de las agrupaciones con menor peso electoral, es decir, a pesar de que 8 partidos políticos obtienen un $5 \%$ del apoyo electoral en las elecciones del 2 de febrero de 2014, un 30\% del total de promesas publicadas por este diario corresponde a esos partidos políticos.

Los lectores de La Teja encontraron pocas promesas electorales en las crónicas o entrevistas: se registran 37 en todo el período.

Conociendo el énfasis informativo y de la cantidad de crónicas publicadas diariamente por medio, no es extraño que La Nación concentre alrededor del $84 \%$ de las promesas divulgadas, el Diario Extra el 13\% y La Teja poco menos del 3\%. Es importante recalcar la capacidad informativa efectiva que tienen los ciudadanos; en este caso hay dos medios de comunicación importantes cuyos lectores - situados en su mayoría en estratos sociales de menor ingreso económico y educativo- potencialmente reciben información reducida de la oferta electoral. Evidentemente, no es culpa de los medios de comunicación, más bien es un llamado a la reflexión crítica en un sistema democrático electoral que tiene entonces que ampliar esas posibilidades de información para el ciudadano.

Por otra parte, agrupando las promesas en temas, los medios La Nación y Diario Extra se aproximan en aquellos de mayor divulgación: política fiscal, infraestructura, educación, empleo, desigualdad/pobreza; solamente que $L a$ Nación le dio mayor preponderancia a promesas de "salud" y Diario Extra a las relativas a "solvencia/eficiencia del Estado".

Se debe señalar la baja frecuencia de promesas en el ámbito de la "seguridad ciudadana", 
que en el caso de La Nación (50) fue mayor que en Diario Extra (2), pero ambas con énfasis en materia penitenciaria. Las promesas en los temas de "tecnología", "telecomunicaciones" y "política exterior" tuvieron una frecuencia muy baja, incluso sumando a los dos medios (3, 3 y 5 , respectivamente).

Los énfasis de las promesas del pac se agrupan en: agricultura (28), educación (27) y política fiscal (27). Por su parte, el pln en ambiente/energía (45), educación (35) e infraestructura (25). Finalmente, el Fa en educación (25), política fiscal (25), salud (22) y ambiente (22).

Para finalizar, se propone posibles temáticas que se podrían abordar en investigaciones futuras:

$\diamond \quad$ Comparación de lo anunciado en el Plan de Gobierno oficial respecto de las promesas, ofertas o compromisos electorales divulgados en medios de comunicación. Esto para detectar promesas no divulgadas en los medios de comunicación $y$ oferta electoral recogida $y$ expuesta por los medios mas no contenida en los programas (ej."el bono alimentario para cubrir las tres comidas diarias en personas en extrema pobreza", prometido por el pLn en enero, a pocas semanas de las elecciones).

$\diamond \quad$ Cumplimiento de la promesa electoral conforme seguimiento de ejercicio de Gobierno del partido político ganador de la contienda electoral, dado que algunas promesas no necesariamente están contempladas en los Planes de Gobierno.

$\diamond \quad$ Profundizar respecto de las preferencias de los medios en destacar ciertas promesas de campaña.

$\diamond \quad$ Nivel de abstracción-concreción de las promesas de campaña, tanto del Programa de Gobierno como de lo divulgado en medios de prensa escrita.

\section{BIBLIOGRAFÍA}

LIBROS

Alcántara, Manuel. Partidos politicos latinoamericanos ¿Instituciones o máquinas ideológicas? México DF, México: Editorial Gernika sa, 2004.

Anduiza, Eva y Bosch, Agustí. Comportamiento político electoral. España: Ariel Ciencia Política, 2004.

Sartori, Giovanni. Aspectos de la democracia. México DF, México: Editorial LimusaWiley sa, 1965.

Stokes, Susan C. Mandates and democracy: neoliberalism by surprise in Latin America. Estados Unidos de América: Cambridge Studies in Comparative Politics, Cambridge University Press, 2001.

\section{PUBLICACIONES PERIÓDICAS}

Aragonès, Enriqueta; Palfrey, Thomas $y$ Postlewaite, Andrew. "Political reputations and campaign promises". Journal of the European Economic Association 4 (5). Nueva Jersey, Eeuu. Wiley-Blackwell, junio 2007: 846-884.

Canel, María José. "Los efectos de las campañas electorales". Revista Comunicación y Sociedad 1(11). Navarra, España. Universidad de Navarra, 1998: 47-67. En: <http://www.unav.es/fcom/ communication-society/es/articulo. php?art_id=150> [Consultado el 12 de enero de 2014].

Hernández, María Aidé. "México: la democracia mexicana, presa de una cultura política con rasgos autoritarios". Revista Mexicana de Sociología 2 (70). México. Universidad Nacional Autónoma de México, abril-junio 2008: 261-303.

Kang, Shin-Goo y Bingham Powell, Jr. "Representation and policy responsiveness: the median voter, election rules, and redistributive welfare spending".The journal of politics 4 (72). Chicago, eevu. University of Chicago Press, octubre 2010: 1014-1028.

Keefer, Philip. "Clientelism, credibility, and the policy choices of young democracies". American Journal of Political Science 4 (51). Nueva Jersey, eeuu. Wiley-Blackwell, octubre 2007: 804-821.

Máiz, Ramón. "Deliberación e inclusión en la democracia republicana". 
Revista Española de Investigaciones Sociológicas (REIS)113. España. Centro de Investigaciones Sociológicas, 2006: 11-47.

Maravall, José María; Przeworski, Adam y Casado, Ma. Teresa. "Reacciones políticas a la economía". Revista Española de Investigaciones Sociológicas (REIS) 87. España. Centro de Investigaciones Sociológicas, 1999: 11-52.

Código Electoral. "Ley 8765”. La Gaceta 171. Costa Rica, 2009.

Diario Extra (2 de octubre de $2013-1^{\circ}$ de febrero de 2014). Todas las ediciones. San José, Costa Rica.

La Nación. Palabra de político. San José, Costa Rica. 2013. En: <http://www.nacion.com/ datafactory/especiales/2013/diciembre/ palabradepolitico> [consultado el 22 de 01 de 2014].

La Nación (2 de octubre de $2013-1^{\circ}$ de febrero de 2014). Todas las ediciones. San José, Costa Rica.

La Teja (2 de octubre de $2013-1^{\circ}$ de febrero de 2014). Todas las ediciones. San José, Costa Rica.
OTROS

Centro de Investigación en Estudios Políticos. Informe de la Encuesta Post-Electoral febrero 2014. Estudios de opinión sociopolítica. Universidad de Costa Rica, 2014.

Programa Estado de la Nación. Decimonoveno Informe Estado de la Nación en Desarrollo Humano Sostenible. San José, Costa Rica: Programa Estado de la Nación, 2013.

Estado de la Nación en Desarrollo Humano Sostenible. Preguntas para la deliberación política. San José, Costa Rica, 2013 En:<http://electoral. estadonacion.or.cr/files/desafiosdhs.pdf> [consultado el 10 de diciembre de 2013].

Instituto Nacional de Estadísticas y Censos (INEc). Información estadística.San José, Costa Rica. En: <http://www.inec.go.cr> [consultado el 13 de diciembre de 2013].

Fecha de ingreso: $22 / 04 / 2014$ Fecha de aprobación: 28/08/2014 